\title{
Magnetotransport and Hall effect studies of $\mathrm{SrRuO}_{3} / \mathrm{SrTiO}_{3}$ superlattices
}

\author{
F. Bern ${ }^{1}$ and M. Ziese ${ }^{1}$ \\ Division of Superconductivity and Magnetism, University of Leipzig, 04103 Leipzig, Germany
}

\begin{abstract}
Good epitaxial growth characteristics make oxide heterostructures of the perovskite family a model system for the study of emerging phenomena at interfaces. Here we report on $\mathrm{SrRuO}_{3} / \mathrm{SrTiO}_{3}$ superlattices as well as single films with individual layer thicknesses between two and 20 unit cells fabricated by pulsed laser deposition. Coherent growth of the interfaces was confirmed by X-Ray and high resolution transmission electron microscopy measurements. Special attention is dedicated to the study of the ordinary and anomalous Hall effect. While $\mathrm{SrRuO}_{3}$ single layers as thin as three unit cells and superlattices with individual layer thicknesses above four unit cells show an anomalous Hall effect similar to the bulk and a temperature-independent high field Hall slope, the ultrathin superlattice shows a temperature dependence in the high field Hall slope and opposite sign in the anomalous Hall effect. Additionally, angle dependent magnetotransport was used to obtain structural information.
\end{abstract}

\section{Introduction}

$\mathrm{SrRuO}_{3}(\mathrm{SRO})$ has received a lot of interest over the past two decades both due to its good growth characteristics and structure that enables it to be used as electrode material in the huge perovskite oxide family. When used in multilayers, new effects may arise due to coupling phenomena at the interface [1] and structural distortion [2], but also new, purely interfacial properties emerge [3]. The SRO/STO interface and structural coupling are of special interest as $\mathrm{SrTiO}_{3}$ is a widely used substrate material in oxide thin film growth and may also serve as insulating barrier in multilayers. Recently, an emerging magnetoelectric effect as well as a magnetic 2DEG [4] has been theoretically predicted.

Bulk SRO exhibits orthorhombic symmetry at room temperature [5], with the lattice parameters $a=5.5670 \AA$, $b=5.5304 \AA$, and $c=7.8446 \AA$. This yields a pseudocubic lattice constant of $a_{p c}=3.93 \AA$, slightly bigger than the STO lattice constant $a_{S T O}=3.905 \AA$. When grown on vicinal (001) STO with the miscut terrace edges along one of the STO crystallographic directions, the SRO [001 $]_{\mathrm{o}}$ axis aligns along the terrace edges [6], the compressive strain leads to a monoclinic distortion with the $[110]_{\mathrm{o}}$ axis slightly off the film normal with an angle of $89.2^{\circ}$ between $\mathrm{a}$ and $\mathrm{b}$ axis, for thin films an additional (triclinic) distortion of $0.012^{\circ}$ between $[001]_{o}$ axis and the ab-plane was reported [7]. Recently, a comprehensive review on SRO thin films was published by Koster et al.[8].

The study of the anomalous Hall effect (AHE) in SRO is of fundamental interest due to several possible scenarios, and received considerable attention in recent years. The AHE denotes the dependence of the transverse resistivity $\rho_{y x}$ on the magnetization $M_{\perp}$ of a conductor as

$$
\rho_{y x}=R_{H} B_{\perp}+R_{S} \mu_{0} M_{\perp},
$$

where $R_{H}$ is the ordinary Hall constant, $B_{\perp}$ the magnetic induction perpendicular to the current direction (which is equal to the applied field $\mu_{0} H$ in the thin film limit), $R_{S}$
Table 1. Samples: layer thicknesses (in unit cells), Curie temperatures, and remanent magnetic moment at $10 \mathrm{~K}$ per Ruthenium atom.

\begin{tabular}{lllc}
\hline Sample & {$[\mathrm{SRO} / \mathrm{STO}]_{n}$} & $\mathrm{~T}_{C}$ & $\mathrm{~m}_{S}\left(\mu_{B} /\right.$ u.c. $)$ \\
\hline F 2/0 & {$[2 / 0]_{1}$} & - & - \\
F 4/0 & {$[4 / 0]_{1}$} & $135 \mathrm{~K}$ & 1.1 \\
F 12/0 & {$[12 / 0]_{1}$} & $140 \mathrm{~K}$ & 1.2 \\
F 100/0 & {$[100 / 0]_{1}$} & $155 \mathrm{~K}$ & 1.7 \\
\hline SL 3/3 & {$[3 / 3]_{15}$} & $85 \mathrm{~K}$ & 0.36 \\
SL 6/9 & {$[6 / 9]_{15}$} & $132 \mathrm{~K}$ & 1.1 \\
SL 12/4 & {$[12 / 4]_{15}$} & $142 \mathrm{~K}$ & 1.4 \\
\hline
\end{tabular}

denotes the anomalous Hall constant and $M_{\perp}$ the magnetization perpendicular to the current direction. While the extrinsic picture predicts a resistivity dependence of $R_{S}$ [9] with a linear negative contribution from skew scattering and a positive quadratic contribution from side jump scattering[10], also a contribution from the Berry phase with a dependence on the crystal structure has been proposed [11]. Recently very good agreement with experimental data has been achieved by a combination of both effects [12].

\section{Experimental}

$\mathrm{SrRuO}_{3}$ films and $\mathrm{SrRuO}_{3} / \mathrm{SrTiO}_{3}$ superlattices (SLs) were fabricated by pulsed laser deposition from stoichiometric polycrystalline targets. Vicinal STO (001) substrates with a small miscut angle of the order of $0.1^{\circ}$, uniform $\mathrm{TiO}_{2}-$ termination and a terrace morphology with typically 150 $400 \mathrm{~nm}$ terrace width were used. Substrate temperature was $650^{\circ} \mathrm{C}$ and oxygen partial pressure 0.14 mbar. The samples studied in this work are listed in table 1 with respective layer thicknesses. Samples are either denoted by the film thickness or by the individual layer thicknesses, both specified by the number of (pseudocubic) unit cells (u.c.). 

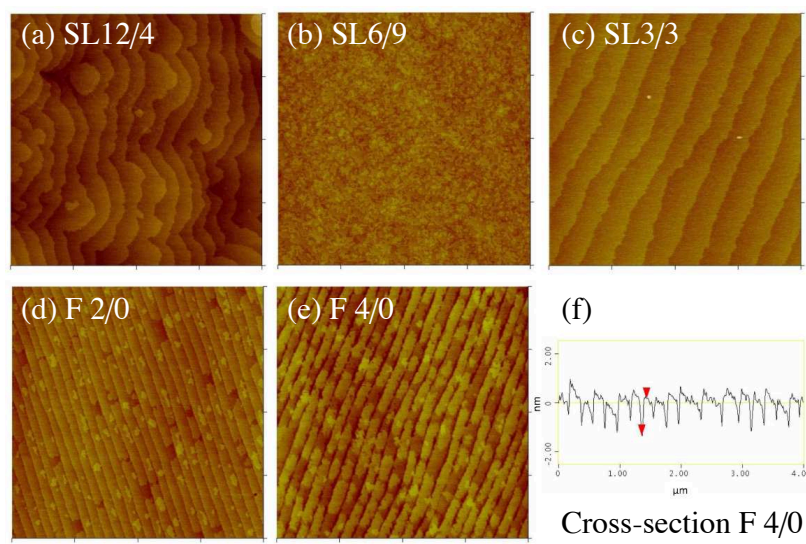

(f)

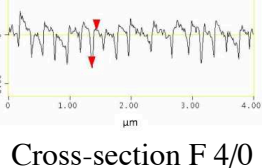

Cross-section F 4/0

Figure 1. (Colour online) AFM images of the superlattices and thinnest films.Scan size is $4 \mu \mathrm{m} \times 4 \mu \mathrm{m}$. In (f) a cross-section analysis for film $4 / 0$ along a line perpendicular to the steps is shown.

Surface morphology was analysed with atomic force microscopy (AFM). Magnetization measurements were performed in a SQUID magnetometer. The diamagnetic signal of the substrate was subtracted from the data. Resistance and Hall-effect measurements were made with a standard four-point technique in van-der-Pauw configuration. The longitudinal and Hall resistivity was calculated taking into account the thickness of the conducting SRO layers only.

\section{Results}

All samples were characterized by AFM measurements. Fig. 1 shows AFM measurements of SLs 12/4, 6/9,3/3 and single films $2 / 0$ and $4 / 0$. In general, the samples showed step-flow growth with the vicinal terraces of the STO substrate clearly to be seen on the sample surface. On the SLs $12 / 4$ and $3 / 3$ adjacent terraces were separated by unit cell high steps. SL6/9 grew partially in island growth mode, probably due to the thicker STO layers. The thin films, however, have a different morphology. As shown in Fig. 1 (d-f) instead of terraces they show one dimensional features along the substrate terraces best described as troughs or valleys. Films $4 / 0$ and $2 / 0$ with thicknesses of about 1.6 and $0.8 \mathrm{~nm}$ have troughs with typical depths of about 1 and $0.5 \mathrm{~nm}$. This means that the troughs do not reach the substrate surface and that the films are continuous. However, they have some tendency towards one dimensional structures with wire widths of about $200(4 / 0)$ and $160 \mathrm{~nm}(2 / 0)$. One might therefore expect an in-plane anisotropy in both transport and magnetic properties. Their formation has been ascribed to $\mathrm{SrO}$ diffusion to the step edges [13]. A close look at the surface of $F 4 / 0$ reveals the formation of islands, such that we have actually a thickness distribution between 3-5 unit cells.

Both, high angle annular dark field-scanning transmission electron microscopy images as well as the observation of superlattice peaks in XRD-scans indicate coherently grown interfaces (not shown). Although some Ru/Ti intermixing at the interface may occur, the SLs clearly maintain the layered structure. Only SL6/9 shows more disorder, as already indicated by the surface morphology.

All samples have their magnetic easy axis in the $(001)_{\mathrm{o}}$ plane at an angle of $\sim 40^{\circ}$ with the film normal for the thicker (F100/0, F12/0, and SL12/4) samples which aligns

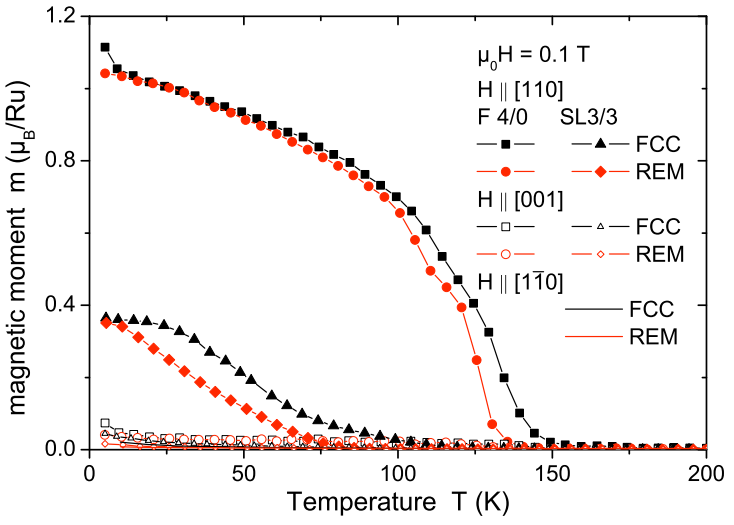

Figure 2. (Colour online) Temperature dependence of the magnetization in $\mu_{B}$ per u.c. perpendicular (filled symbols) to the film as well as in-plane (open symbols) of F4/0 and SL3/3. Black symbols refer to cooling with an applied magnetic field of $0.1 \mathrm{~T}$, red symbols show the remanence behaviour during heating of the sample with no external field applied.

with the film normal in the thin samples [14], see Fig. 2. One might attribute this effect to twinning, but then again, the coercive field increases for decreasing layer thickness indicating a higher anisotropy. Only the thinnest film F2/0 does not show magnetic order and SL3/3 has a considerably reduced Curie temperature (see Fig. 2, and Tab. 1), which can be explained considering finite-size scaling effects [15], [16].

The temperature dependence of the resistivity is shown for all samples studied in Fig. 3. Only the thinnest samples do

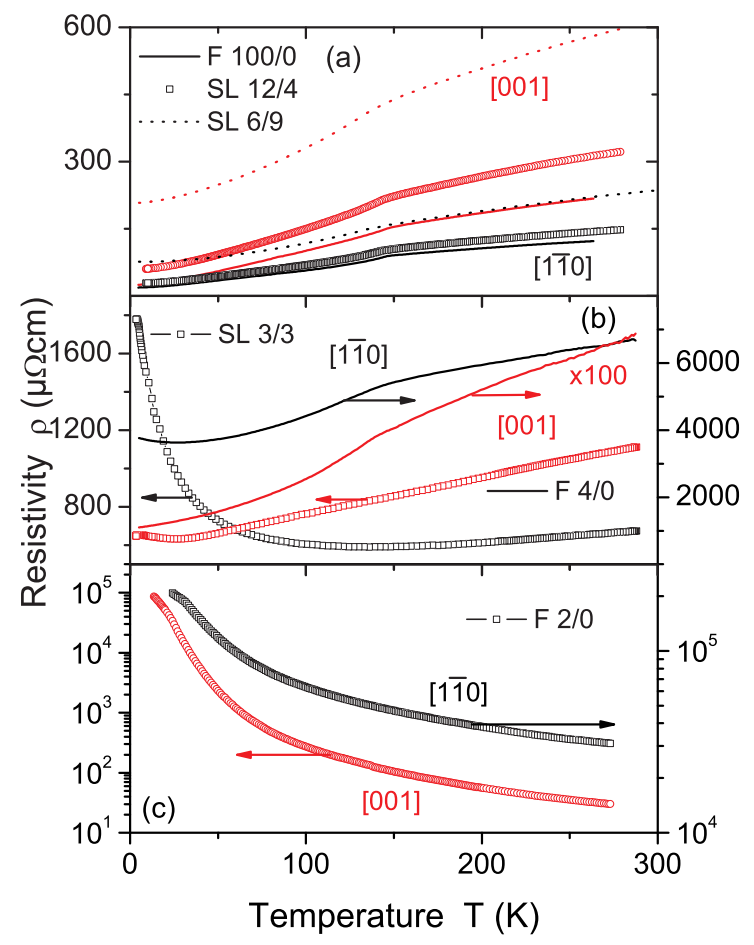

Figure 3. (Colour online) Temperature dependence of the resistivity of all the samples studied. Arrows indicate the respective scale. The black curves give the resistivity along the $[1 \overline{1} 0]_{\mathrm{o}}$, the red ones along the $[001]_{0}$ direction. Samples with more than 5 u.c. thick individual layers in (a), 3-4 u.c. thick in (b) and (c) with 2 u.c. 


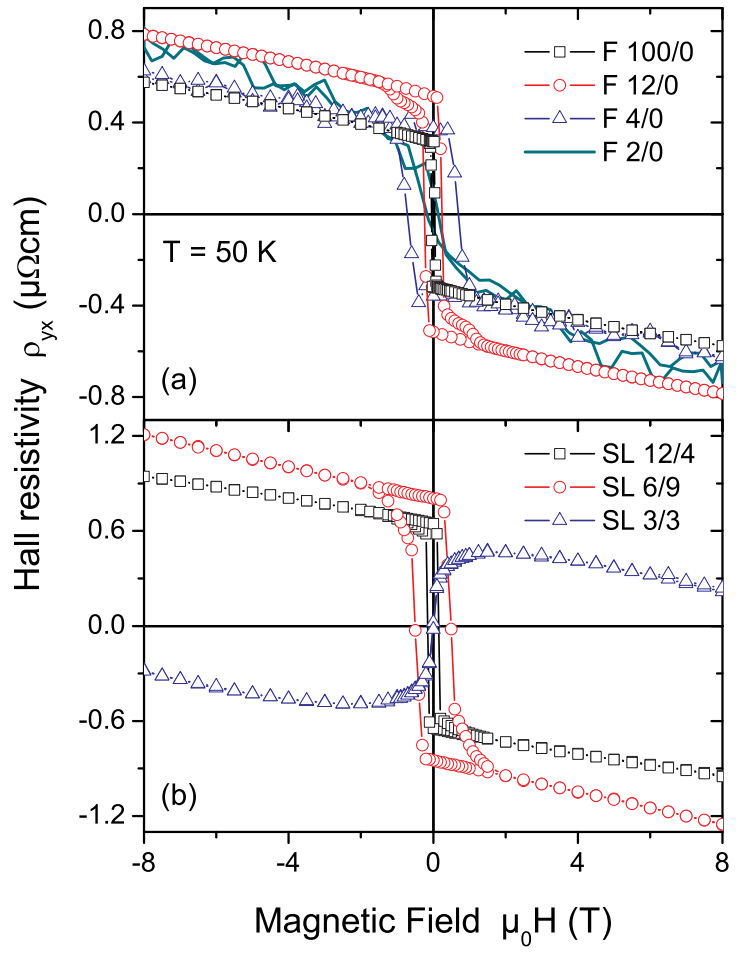

Figure 4. (Colour online) $\rho_{y x}$ for all samples at $50 \mathrm{~K}$. In (a) the thin films are shown, wheras (b) depicts the superlattices.

not show the characteristic slope change at $T_{\mathrm{C}}$, instead they show a pronounced increase of the resistivity with decreasing temperature. As can be seen in Fig 3(c), the thinnest sample has a negative temperature coefficient in the whole range studied. All samples show a distinct anisotropy in the

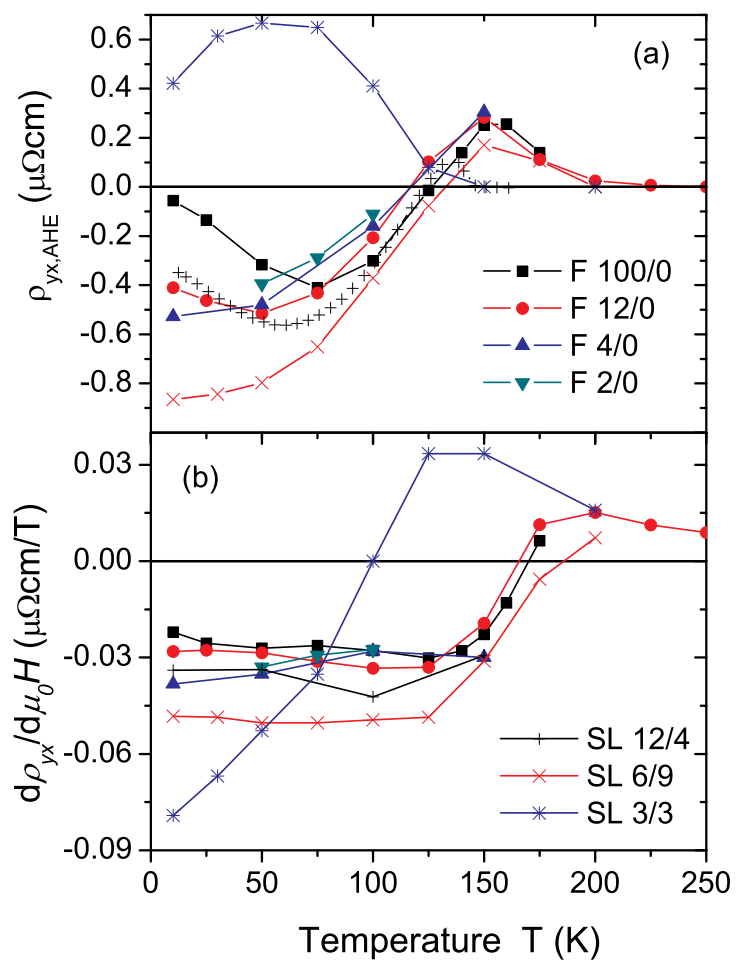

Figure 5. (Colour online) Temperature dependence of the Hall coefficients. In (a) the anomalous Hall contribution $\rho_{y x, A H E}$ is shown, (b) $d \rho_{y x} / d \mu_{0} H$ determined from the high field slope.

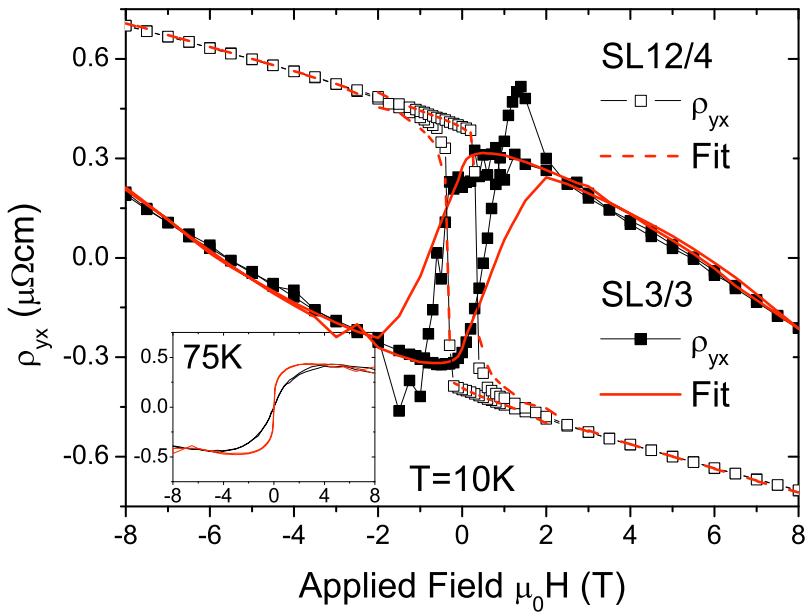

Figure 6. (Colour online) Hall resistivity vs. field at 10K. Square symbols show the measured $\rho_{y x}$, lines are fits based on magnetization measurements according to Eq. 1. Filled symbols refer to SL $3 / 3$, open symbols to SL6/9. The inset shows a fit at $T=75 \mathrm{~K}$ of $\mathrm{SL} 3 / 3$.

transport properties, indicating also crystalline asymmetry in plane. Another striking feature is the change of direction of minimum resistivity, which is parallel to $[1 \overline{1} 0]_{\mathrm{o}}$ for thick films and to $[001]_{\mathrm{o}}$ for individual layer thickness below 6 u.c (Fig 3(b),(c)). This is probably correlated to the above mentioned trough-like features observed on the surface (see Fig. 1).

Fig 4 shows the Hall resistivity vs. applied field at $50 \mathrm{~K}$. The thin films show comparable behaviour besides having different coercive fields. The bigger $\rho_{y x, A H E}$ for F12/0 can be understood as due to higher resistivity (as compared to F100/0) or higher magnetization (as compared to the thinnest films). Although exhibiting no measurable magnetic moment F2/0 clearly shows an anomalous Hall contribution. The thick SLs show a similar behaviour as the films, however, SL3/3 is strikingly different showing a reversed sign of $\rho_{y x, A H E}$. The temperature dependence of the two Hall coefficients as depicted in Fig. 5 reveals that this positive $\rho_{y x, A H E}$ of SL3/3 is maintained over the whole temperature range studied. Further, a strong temperature dependence of $d \rho_{y x} / d \mu_{0} H$ for SL3/3 with a sign change close to $T_{\mathrm{C}}$ is observed. The other samples show behaviour similar to those reported in literature [12], [17]. Note that the high field slope in Fig. 5(b) is not equal to the Hall constant $R_{H}$ for temperatures above $T_{C}$, but contains a nonlinear contribution from $\rho_{y x, A H E}$ as the magnetization is not saturated in the field range up to 8T. In Fig. 6 we fitted the Hall data with the recorded magnetization data according to Eq.1. While this works perfectly for the thick SLs there are deviations for SL3/3 during the magnetization reversal process. For higher temperatures the fit of SL3/3 was impossible, an example curve at $75 \mathrm{~K}$ is shown in the inset. This indicates a nonlinear dependence of $R_{S}$ on the magnetization in the observed anomalous Hall effect.

To gain further insight into structural symmetry of the samples, as this might be correlated to the Berry-phase contribution to $\rho_{y x, A H E}$ [11], we performed angular dependent magnetoresistance measurements [18]. SLs 12/4 and 6/9 in Fig. 7 show clearly an orthorhombic symmetry. The easy axis is considerably closer to the substrate normal for SL6/9 indicating a stronger monoclinic distortion. The sin- 


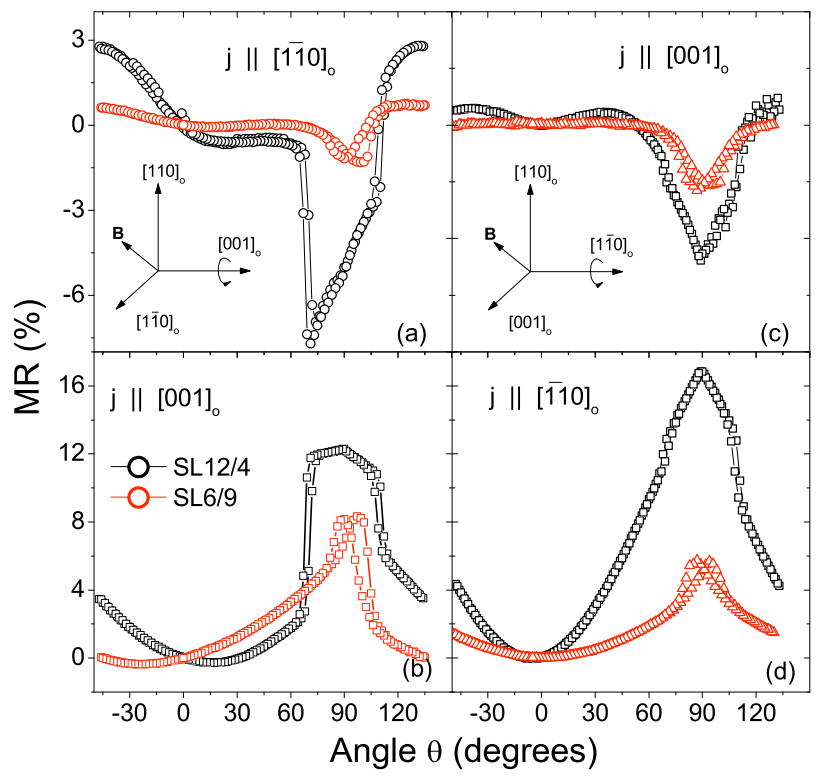

Figure 7. (Colour online) Angle dependence of the magnetoresistance for rotation in the $(001)_{\mathrm{o}}$ plane $((\mathrm{a}),(\mathrm{c}))$ and $(1 \overline{1} 0)_{\mathrm{o}}$ plane ((b),(d)) of the SL12/4 (squares) and 6/9 (triangles) at 10K.

gle film F4/0 in Fig. 8 shows the continuation of the trend towards uniaxial magnetic anisotropy normal to the film plane maintaining however anisotropy in transport. The angular MR of SL3/3 in Fig. 8 looks distinctly different indicating absence of orthorhombic order. However, the strong anisotropy in transport may hide the structural features.

\section{Conclusions}

Substrate induced compressive strain rotates the magnetic easy axis away from the $\mathrm{b}$ axis towards the substrate normal, while in transport anisotropy in the film plane is conserved. SLs with thicker individual layers as well as single films show a Hall effect similar to the ones reported in literature. The thinnest superlattice SL3/3 shows a completely different behaviour, a positive anomalous Hall effect as normally only observed just below $T_{C}$ in SRO. Furthermore, the anomalous Hall contribution $\rho_{y x, A H E}$ cannot be simply related to the magnetization. Further experimental and theoretical work is required to elucidate the observed effect.

\section{Acknowledgements}

This work was supported by the DFG within SFB 762 "Functionality of Oxide Interfaces"; F. B. is member of the Leipzig School of Natural Sciences "BuildMoNa". We gratefully acknowledge the help of I. Vrejoiu in providing samples and AFM images, and for discussion. We thank E. Pippel and D. Hesse for HR-TEM images, and A. Setzer for XRD measurements.

\section{References}

1. M. Ziese, I. Vrejoiu, E. Pippel, P. Esquinazi, D. Hesse, C. Etz, J. Henk, A. Ernst, I.V. Maznichenko, W. Hergert et al., Phys. Rev. Lett. 104, 167203 (2010)

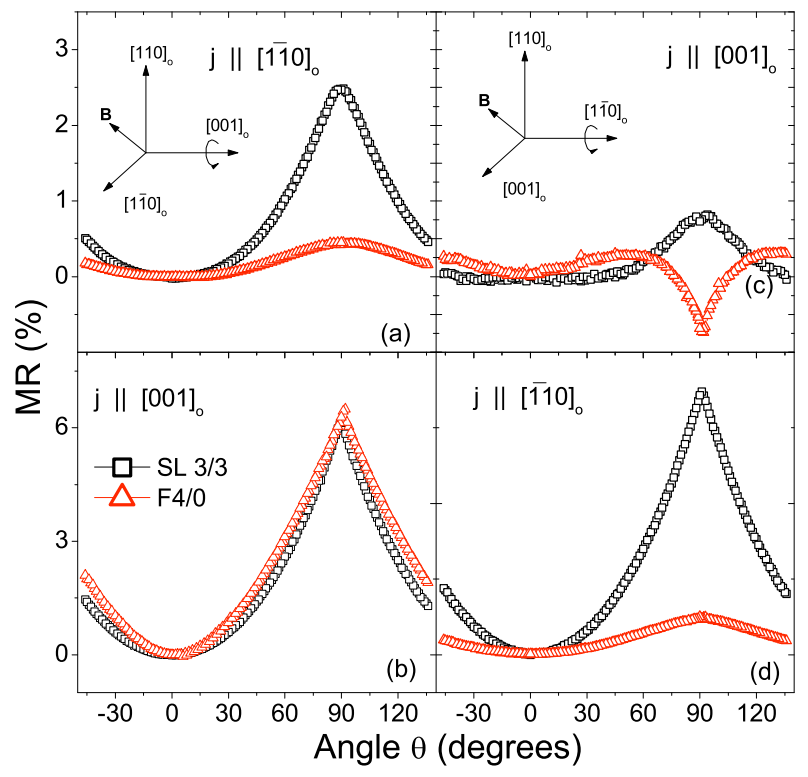

Figure 8. (Colour online) Angle dependence of the magnetoresistance for rotation in the $(001)_{\mathrm{o}}$ plane $((\mathrm{a}),(\mathrm{c}))$ and $(1 \overline{1} 0)_{\mathrm{o}}$ plane ((b),(d)) of the SL3/3 (squares) and F4/0 (triangles) at 50K.

2. M. Ziese, I. Vrejoiu, Phys. Rev. B 84, 104413 (2011)

3. A. Ohtomo, H.Y. Hwang, Nature (London) 427, 423 (2004)

4. M. Verissimo-Alves, P. García-Fernández, D.I. Bilc, P. Ghosez, J. Junquera, Phys. Rev. Lett. 108, 107003 (2012)

5. C.W. Jones, P.D. Battle, P. Lightfoot, W.T.A. Harrison, Acta Crystallographica Section C 45, 365 (1989)

6. A. Vailionis, W. Siemons, G. Koster, Appl. Phys. Lett. 91, 071907 (2007)

7. D. Kan, Y. Shimakawa, Cryst. Growth Des. 11, 5483 (2011)

8. G. Koster, L. Klein, W. Siemons, G. Rijnders, J. Dodge, C. Eom, D. Blank, M. Beasley, Rev. Mod. Phys. 84, 253 (2012)

9. Y. Kats, I. Genish, L. Klein, J.W. Reiner, M.R. Beasley, Phys. Rev. B 70, 180407(R) (2004)

10. M. Izumi, K. Nakazawa, Y. Bando, Y. Yoneda, H. Terauchi, J. Phys. Soc. Japan 66, 3893 (1997)

11. Z. Fang, N. Nagaosa, K.S. Takahashi, A. Asamitsu, R. Mathieu, T. Ogasawara, H. Yamada, M. Kawasaki, Y. Tokura, K. Terakura, Science 302, 92 (2003)

12. N. Haham, Y. Shperber, M. Schultz, N. Naftalis, E. Shimshoni, J.W. Reiner, L. Klein, Phys. Rev. B 84, 174439 (2011)

13. R. Bachelet, F. Sánchez, J. Santiso, C. Munuera, C. Ocal, J. Fontcuberta, Chemistry of Materials 21, 2494 (2009)

14. M. Schultz, S. Levy, J. Reiner, L. Klein, Phys. Rev. B 79, 125444 (2009)

15. M. Izumi, K. Nakazawa, Y. Bando, J. Phys. Soc. Japan 67, 651 (1998)

16. M. Izumi, K. Nakazawa, Y. Bando, Y. Yoneda, Solid State Ionics 108, 227 (1998)

17. R. Mathieu, C.U. Jung, H. Yamada, A. Asamitsu, M. Kawasaki, Y. Tokura, Phys. Rev. B 72, 064436 (2005)

18. M. Ziese, I. Vrejoiu, D. Hesse, Phys. Rev. B 81, 184418 (2010) 